\title{
Expert Knowledge Correlated Intrusion Detection System Evaluation Framework for Heterogeneous loT
}

This paper was downloaded from TechRxiv (https://www.techrxiv.org).

LICENSE

CC BY-NC-SA 4.0

SUBMISSION DATE / POSTED DATE

02-10-2021 / 18-11-2021

\section{CITATION}

A, Nitish; Hanumanthappa, J.; S.P, Shiva Prakash; Krinkin, Kirill (2021): Expert Knowledge Correlated Intrusion Detection System Evaluation Framework for Heterogeneous loT. TechRxiv. Preprint. https://doi.org/10.36227/techrxiv.16722784.v2

$\mathrm{DOI}$

10.36227/techrxiv.16722784.v2 


\title{
Expert Knowledge Correlated Intrusion Detection System Evaluation Framework for Heterogeneous IoT
}

\author{
Nitish $\mathrm{A}^{1 \dagger}$, Hanumanthappa $\mathrm{J}^{1 \dagger}$, Shiva Prakash S. $\mathrm{P}^{2 * \dagger}$ \\ and Kirill Krinkin ${ }^{3 \dagger}$
}

${ }^{1}$ Department of Studies in Computer Science, University of Mysore, Manasagangothri, Mysuru, 570006, Karnataka, India.

${ }^{2}$ Department of Information Science and Engineering, JSS

Science and Technology University, JSS Technical Institutions

Campus, Mysuru, 570006, Karnataka, India.

${ }^{3}$ Department of Software Engineering and Computer

Applications, St. Petersburg Electrotechnical University "LETI",

Ulitsa Professora Popova, 5, St. Petersburg, 197022, Russia.

\author{
*Corresponding author(s). E-mail(s): shivasp@sjce.ac.in; \\ Contributing authors: nitish.anantha@acm.org; \\ hanums_j@yahoo.com; kirill@krinkin.com; \\ †These authors contributed equally to this work.
}

\begin{abstract}
The dynamic contexts of heterogeneous Internet of Things (HetIoT) adversely affect the performance of learning-based network intrusion detection systems (NIDS) resulting in increased misclassification ratesnecessitating an expert knowledge correlated evaluation framework. The proposed generalizable framework includes intrusion root cause analysis, correlation model, and correlated classification metrics that can be generalized over any NID dataset, corresponding expert knowledge, detection technique, and learning-based algorithm to facilitate contextawareness in reducing false alerts. To achieve this, we perform experimentations on the Bot-IoT dataset - with generalized traffic behaviors from multiple existing NID datasets - employing the Support Vector Machine (SVM) machine learning and Multilayer Perceptron (MLP) shallow neural network classifiers, demonstrating the generalizability,
\end{abstract}


robustness, and improved performance of the propounded framework compared to the existing literature. Furthermore, the proposed framework offers minimal processing overhead on the classifier algorithms.

Keywords: Context-awareness, Correlated evaluation metrics, Expert knowledge correlation, Heterogeneous IoT, Network intrusion detection framework, Root cause analysis

\section{Introduction}

Heterogeneous networks that offer ad hoc network-like flexibility and IoT network-like connectivity are suitable for ubiquitous computing and proliferating content consumption in critical applications across domestic and industrial space [1]-[9]. Such networks are called heterogeneous IoT (or HetIoT) [13].

Learning-based network intrusion detection systems (NIDS) for such HetIoT infrastructures suffer from high misclassification rates due to contextunaware statistical evaluation of (machine or deep) learning-based algorithms as HetIoT involves dynamic contexts [1], [25]. Such dynamic scenarios necessitate reinforcing an expert knowledge (EK) framework for context-aware decision-making and minimizing false alerts [5], [11], [15], [16] to avoid high recovery costs due to failure. EK can include information like high-level traffic behaviors, constituting device specifications, operating conditions, and human feedback.

We propose a generalized EK correlated evaluation framework with correlated metrics that performs well on imbalanced data and can supplement any (machine or deep) learning-based algorithm to obtain reduced false alerts for any intrusion detection (ID) application, provided a relevant expert knowledgebase for the network traffic dataset is available. The generalizability of the propounded framework is achieved by performing classification on the Bot-IoT dataset [9], which contains the traffic characteristics of IoT devices in addition to those from the existing NID datasets. Furthermore, this framework permits policy distribution across the HetIoT subnets for context-specific intrusion detection and prevention.

We evaluate our proposition on the SVM and MLP classifier algorithms by employing the Bot-IoT dataset [9] and constructing a relevant expert knowledgebase with the following contributions required for a generalized heterogeneous NIDS framework:

1. A high-level EK framework (RCA table) for intrusion root cause analysis.

2. A reinforcement method (Correlation algorithm) for correlating ID alerts with EK inferences for reducing false alerts.

3. Correlated evaluation metrics derived from standard ID evaluation metrics based on the Correlation algorithm. 
Table 1 Notations used in the paper and their description

\begin{tabular}{|c|c|}
\hline Notation & Description \\
\hline$D_{M \times N}=\left\{d_{t}, y_{t}\right\}$ & $\begin{array}{l}\text { Network traffic dataset having } M \text { samples and } \\
N \text { features with class labels }\end{array}$ \\
\hline $\mathbf{P}(f(d))$ & Misuse detection prediction \\
\hline$C_{i} \in C$ & $C_{i}=\{0,1\}$ binary class labels \\
\hline$E_{R C A}(k)$ & $k^{t h} \mathrm{RCA}$ expert knowledge instance \\
\hline $\mathcal{E}\left(d_{q}, k_{r}\right)$ & Evaluation function \\
\hline$\xi, \xi_{F P}, \xi_{F N}$ & RCA EK error, FP error, FN error \\
\hline $\mathcal{A}, \mathcal{P}, \mathcal{R}, \mathcal{F}$ & $\begin{array}{l}\text { Correlated classification metrics - Accuracy, Precision, } \\
\text { Recall, and F1-score }\end{array}$ \\
\hline $\mathcal{T}$ & Time complexity \\
\hline $\mathbb{F}: \mathcal{G}(\mathrm{D}, \mathrm{E}, \mathrm{ID}, \mathrm{A})$ & $\begin{array}{l}\text { NIDS function generalized over NID dataset, EK, } \\
\text { ID technique, and algorithm }\end{array}$ \\
\hline
\end{tabular}

4. Extensive experimentations on the Bot-IoT dataset generalized traffic behaviors using SVM and NLP classifiers - demonstrating the contextawareness, robustness and generalizability of the propounded HetIoT-NIDS framework.

Table 1 provides a list of symbols used in the paper and their description.

\section{Related Work}

The existing NIDS policies for heterogeneous networks [1], [6]-[12] suffer from high misclassification rates due to context-unaware traffic processing by learning-based algorithms, adversely affecting the HetIoT performance.

Researchers have proposed means to reduce misclassifications involving threat context computation [11] and intrusion root cause analysis [25]. However, such works employ ontological techniques [16] that are computationheavy [1] and provide no information on the decentralization of policies, affecting the context-awareness of HetIoT-NIDS especially during big-data processing.

We present a comparative summary of the existing heterogeneous NIDS schemes in Table 2 with respect to the proposed decentralization $(D e c)$, heterogeneity $(\mathrm{Het})$, context-awareness $(C A)$, and generalizability $(\mathcal{G})$ parameters.

\section{Correlated Evaluation Framework}

The dynamic contexts in a HetIoT adversely affect the context unaware lowlevel statistical inferences of ID algorithms. Thus, reinforcing them with the extracted high-level behavioral descriptions improves network redesign that is context-aware and resistant to frequent security compromises, benefitting from the interpretability of knowledge-driven specification and the adaptability of data-driven detection [1]. To achieve this, we propose an EK framework. 
Table 2 Comparative summary of existing heterogeneous NIDS schemes with the proposed framework parameters

\begin{tabular}{lllll}
\hline Scheme & Dec & Het & CA & $\mathcal{G}$ \\
\hline$[2]$ & $x$ & $\checkmark$ & $x$ & D \\
{$[3]$} & $x$ & $\checkmark$ & $\checkmark$ & D, A \\
{$[4]$} & $x$ & $\checkmark$ & $x$ & D \\
{$[5]$} & $x$ & $\checkmark$ & $x$ & D \\
{$[6]$} & $\checkmark$ & $\checkmark$ & $x$ & D \\
{$[7]$} & $x$ & $x$ & $x$ & D, A \\
{$[8]$} & $x$ & $\checkmark$ & $x$ & D, E, A \\
{$[9]$} & $x$ & $\checkmark$ & $x$ & D, A \\
{$[10]$} & $\checkmark$ & $x$ & $x$ & D, ID, A \\
{$[11]$} & $x$ & $\checkmark$ & $\checkmark$ & - \\
{$[12]$} & $x$ & $\checkmark$ & $x$ & D, ID, A \\
{$[16]$} & $\checkmark$ & $\checkmark$ & $\checkmark$ & - \\
Proposed & $\checkmark$ & $\checkmark$ & $\checkmark$ & D, E, ID, A \\
work & & & & \\
\hline
\end{tabular}

\subsection{Root Cause Analysis Expert Knowledge Framework}

The proposed root cause analysis expert knowledge (RCA EK) framework offers a high-level intuition for identifying faults in the HetIoT behavior for a threat context with potential attacks and analyzes root causes of the intrusions encountered. Such a setting is helpful for easier network maintenance, as it reduces frequently examining network logs. Moreover, interpreting network logs from heterogeneous devices is never straightforward and requires additional computations and time to normalize the metadata information from various sources.

Table 3 presents a sample of the proposed attack-wise RCA expert knowledgebase. The detailed version is presented in the Appendix section.

\subsection{Misuse Detection Model}

Misuse detection classifies the network traffic instances according to the class labels.

Definition 1 (Misuse Detection). Let $D=\left\{d_{t}, y_{t}\right\}_{t=1}^{M}$ be the data-driven information (dataset). The prediction (binary classification) that an instance belongs to an attack class $C_{i} \in C$ where $C=\{0,1\}$ is,

$$
\mathbf{P}\left(f\left(d_{t}\right)=1 \mid C_{i}\right)
$$

We employ the proposed misuse detection model using the SVM and MLP classifiers. 


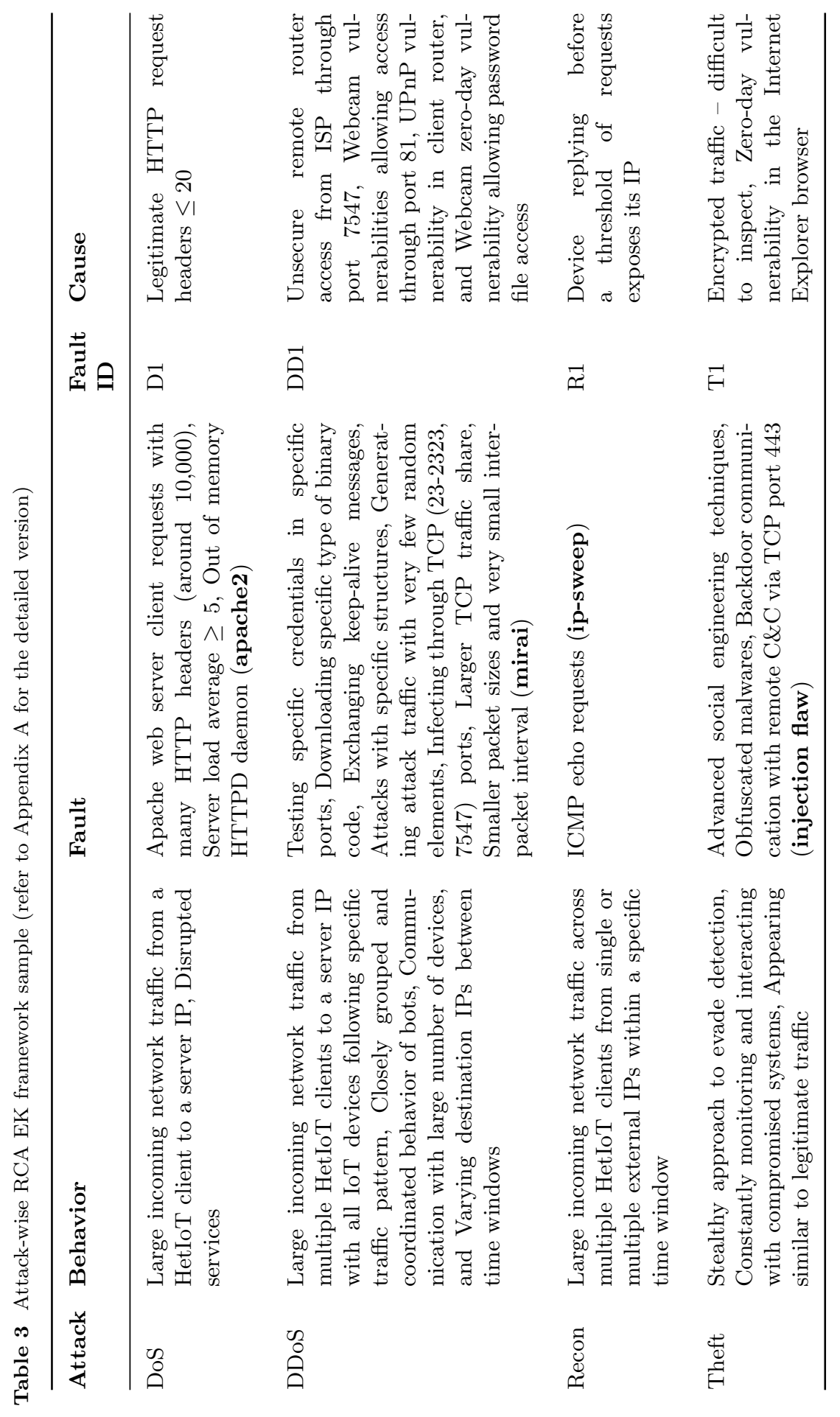




\subsection{Correlation Model}

The Correlation model correlates the ID alerts from the classifier algorithms with the EK inferences from the RCA table.

Definition 2 (Correlation). Let $E_{R C A}(k) \forall k \in K$ be an expert knowledge instance of a dataset instance $d \in D$. Let $\mathcal{E}:(d, k) \rightarrow C$ be an evaluation function such that, evaluating the dataset and the corresponding expert knowledge instances helps detect an attack label instance. i.e.,

$$
\mathcal{E}\left(d_{q}, k_{r}\right)=C_{i}
$$

Therefore, the ID alerts and the corresponding EK inferences are correlated (i.e., yield same class labels) iff,

$$
\mathbf{P}\left(f\left(d_{q}\right)\right) \longleftrightarrow E_{R C A}\left(k_{r}\right)
$$

\subsubsection{Correlation Algorithm}

We employ the proposed Correlation model in Algorithms 1 and 2 to correlate the predicted alerts obtained from the learning-based classifiers. Algorithm 1 - analogous to equation (3) depends on the Algorithm 2, which is analogous to equation (2). The Correlation algorithm is generic and is independent of learning-based algorithms, NID datasets and corresponding EK, and ID techniques.

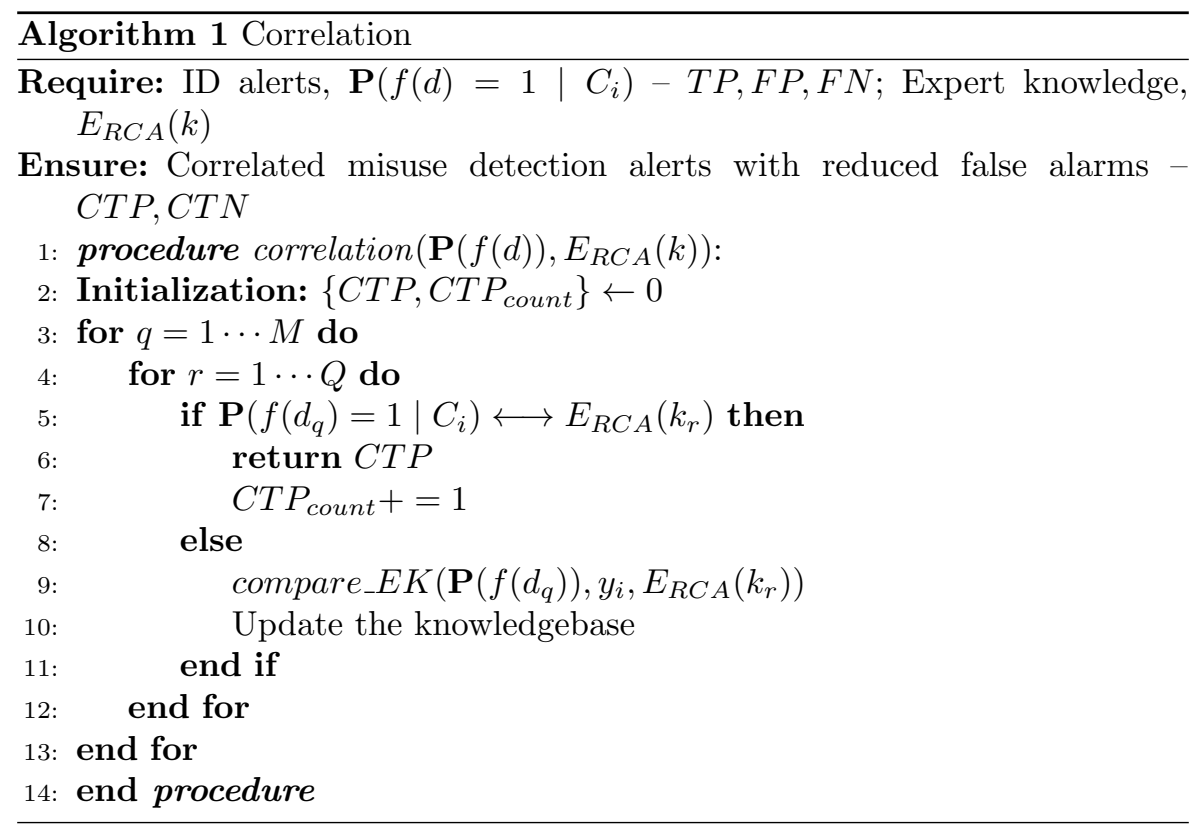



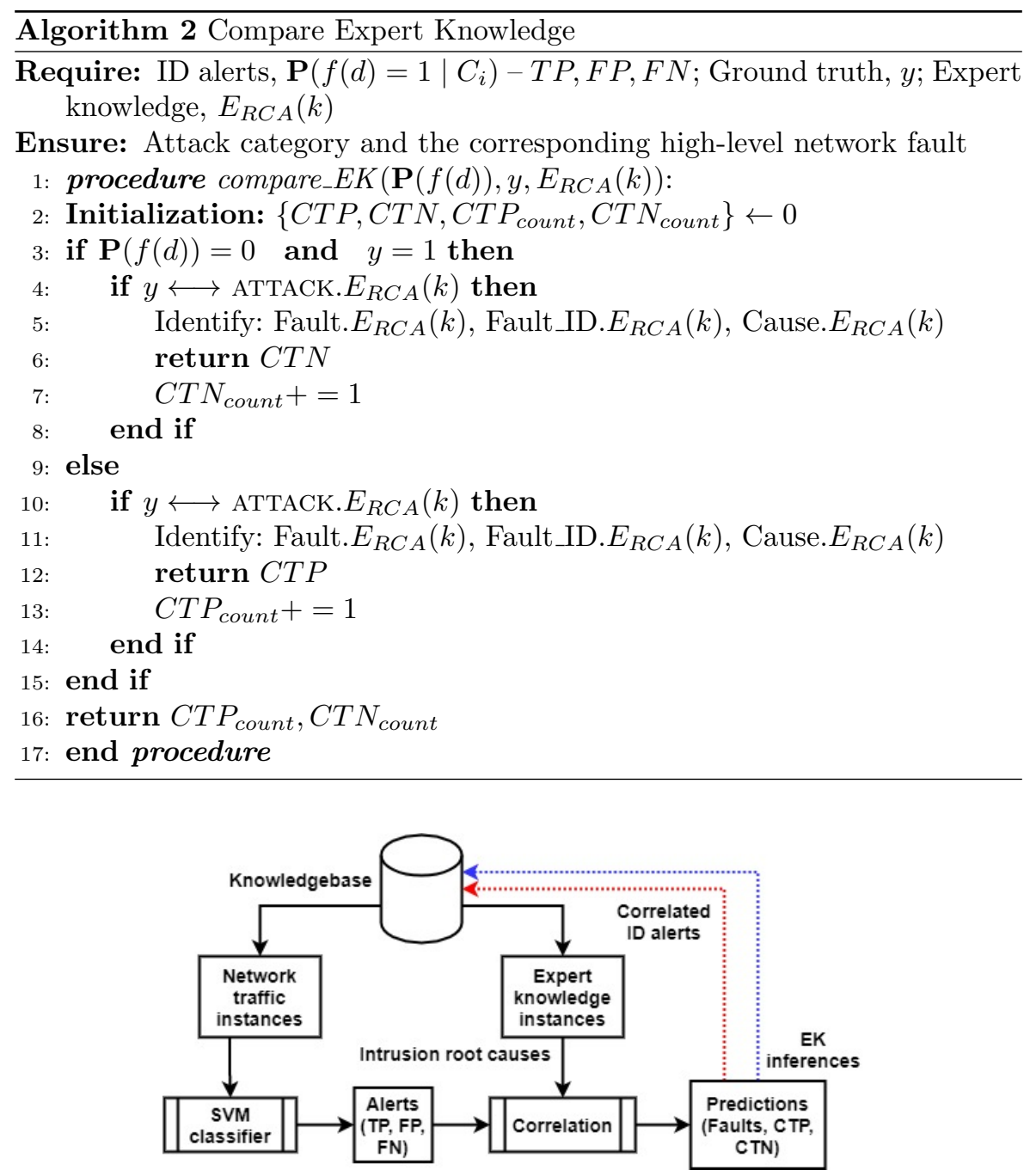

Fig. 1 Proposed correlated HetIoT-NIDS architecture

The correlated alerts with labels and the EK inferences are updated to the knowledgebase - as in Fig. 1. Finally, the Correlation algorithm outputs the alerts described in Table 4.

\subsubsection{Correlated Intrusion Detection Metrics}

We formulate the correlated ID metrics from the results of Correlation algorithm. We derive the proposed metrics from the standard classification metrics of a learning-based algorithm.

The correlation error $\xi$ due to inefficiency in preparing the RCA table $E_{R C A}$ (i.e., unavailability of corresponding EK instance for an ID attack instance) 
8 Expert Knowledge Correlated IDS Evaluation Framework for HetIoT

Table 4 Standard ID alerts and their correlations

\begin{tabular}{ll}
\hline Classification Alerts & Correlated Alerts \\
\hline True Positive $(T P)$ & Correlated True Positive $(C T P)$ \\
True Negative $(T N)$ & - \\
False Positive $(F P)$ & Correlated True Negative $(C T N)$ \\
False Negative $(F N)$ & Correlated True Positive $(C T P)$ \\
\hline
\end{tabular}

results in missed false ID alerts $(F N$ and $F P)$-reducing the metric scores.

$$
\xi=\xi_{F P}+\xi_{F N}
$$

Such misclassifications upon further examination lead to new HetIoT behaviors updated in the expert knowledgebase, gradually reducing the error. i.e.,

$$
\begin{aligned}
&\left(\xi_{F P}, \xi_{F N}\right) \downarrow 0 \\
& \Rightarrow \xi \downarrow 0
\end{aligned}
$$

Therefore, we formulate the correlated ID evaluation metrics as follows:

- Correlated Accuracy $(\mathcal{A})$ :

$$
\begin{aligned}
\text { Accuracy } & =\frac{T P+T N}{T P+T N+F P+F N} \\
\Rightarrow \mathcal{A} & =\frac{C T P+T N}{C T P+T N+C T N+\xi}
\end{aligned}
$$

- Correlated Precision $(\mathcal{P})$ :

$$
\begin{aligned}
\text { Precision } & =\frac{T P}{T P+F P} \\
\Rightarrow \mathcal{P} & =\frac{C T P}{C T P+C T N+\xi_{F P}}
\end{aligned}
$$

- Correlated Recall $(\mathcal{R})$ :

$$
\begin{aligned}
\text { Recall } & =\frac{T P}{T P+F N} \\
\Rightarrow \mathcal{R} & =\frac{C T P}{C T P+\xi_{F N}}
\end{aligned}
$$


- Correlated F1-score $(\mathcal{F})$ :

$$
\begin{aligned}
F 1-\text { score } & =2 \times \frac{\text { Precision } \times \text { Recall }}{\text { Precision }+ \text { Recall }} \\
\Rightarrow \mathcal{F} & =2 \times \frac{\mathcal{P} \times \mathcal{R}}{\mathcal{P}+\mathcal{R}}
\end{aligned}
$$

\section{Algorithm Analysis}

We perform formal analyses of the Correlation algorithm to explore its properties and prove that it has a lower computational overhead.

Theorem 1 (Time Complexity of Correlation Algorithm). For $M$ traffic instances and $Q$ expert knowledge instances, the worst-case time complexity of the Correlation algorithm is

$$
\mathcal{T}_{\text {correlation }} \rightarrow \mathcal{O}(M Q)
$$

Proof The proposed correlation framework constitutes the correlation and compare_EK procedures in Algorithms 1 and 2, respectively.

Algorithm 2 begins with an initialization instruction $I_{1}$ (in line 2) with constant time, followed by a conditional instruction (either if $I_{2}$ in line 3 or else $I_{3}$ in line 9) with a total of two instructions. Four additional instructions $I_{21}-I_{24}$ (in lines $4-7$ ) follow $I_{2}$, each with constant time. Similarly four instructions $I_{31}-I_{34}$ (in lines $10-13$ ) follow $I_{3}$. Finally, a return instruction $I_{4}$ (in line 16) is executed in constant time. Therefore, the time complexity of Algorithm 2 is as follows:

$$
I_{\text {compare_EK }} \leftarrow\left\{\begin{array}{l}
I_{1}+I_{2}+I_{21}+I_{22}+I_{23}+I_{24}+I_{4} \\
I_{1}+I_{3}+I_{31}+I_{32}+I_{33}+I_{34}+I_{4}
\end{array}\right.
$$

Any one of the above paths is executed with a total of seven instructions in constant time.

Algorithm 1 correlates ID alerts with corresponding EK inferences for a matched attack category in each instance. It begins with an initialization instruction $I_{1}$ (in line 2) with constant time, followed by a conditional instruction (either if $I_{2}$ in line 5 or else $I_{3}$ in line 8), totaling two instructions executing $M Q$ times. $I_{2}$ is followed by two more instructions $I_{21}$ and $I_{22}$ (in lines 6 and 7 ) with constant times and $I_{3}$ is followed by a function call to Algorithm 2 with $I_{\text {compare_EK }}$ time and an update instruction $I_{31}$ (in lines 9 and 10), totaling nine instructions with constant time. Therefore, the time complexity of the Correlation algorithm is as follows:

$$
I_{\text {correlation }} \leftarrow\left\{\begin{array}{l}
I_{1}+M Q \times\left(I_{2}+I_{21}+I_{22}\right) \\
I_{1}+M Q \times\left(I_{3}+I_{\text {compare_EK }}+I_{31}\right)
\end{array}\right.
$$

The above execution paths contain $1+3 \times M Q$ and $1+9 \times M Q$ instructions, respectively.

Hence, it is clear from the above equations that the worst-case time complexity of the Correlation algorithm involves executing $1+9 \times M Q$ instructions at most. Therefore, the time complexity of the proposed Correlation algorithm is, 


$$
\mathcal{T}_{\text {correlation }} \rightarrow \mathcal{O}(M Q)
$$

Corollary 1 (Average Correlations). The average correlations of the Correlation algorithm linearly reduce to

$$
\mathcal{T}_{\text {correlation }} \rightarrow \mathcal{O}(M)
$$

Proof Let $D=\left\{d_{i}, y_{i}\right\}_{i=1}^{M} \in \mathbb{R}^{M \times N}$ be the network traffic dataset and $E_{R C A}=$ $\left\{k_{j}, y_{j}\right\}_{j=1}^{Q} \in \mathbb{R}^{Q \times 5}$ be the corresponding expert knowledgebase for a matching attack category $C$.

For a sufficiently large network traffic dataset, the corresponding expert knowledgebase is comparatively very small - as a large number of the dataset instances refer to a very small set of EK instances with a matching attack category in a threat context. Hence, we have

$$
D_{(M \times N)}>>E_{R C A_{(Q \times 5)}}
$$

because $\mathbb{R}^{Q \times 5}<<\mathbb{R}^{M \times N}$. Hence $Q$ becomes negligible against $M$ on an average.

Therefore, the average-case time complexity of the Correlation algorithm is

$$
\mathcal{T}_{\text {correlation }} \rightarrow \mathcal{O}(M)
$$

We introduce the Generalizability property as a part of the proposed evaluation framework.

Definition 3 (Generalizability). Let $\mathbb{F}$ be an NIDS function accommodating a dataset $\mathrm{D}$, corresponding expert knowledgebase $\mathrm{E}$, intrusion detection technique ID, and corresponding algorithm $\mathrm{A}$. The Generalizability of $\mathbb{F}$ is

$$
\mathbb{F}: \mathcal{G}(\mathrm{D}, \mathrm{E}, \mathrm{ID}, \mathrm{A})
$$

Since the Correlation model utilizes context-specific expert knowledge from a network traffic data of any attack type, it can supplement any (machine or deep) learning-based algorithm for supervised misuse detection or unsupervised anomaly detection, provided a suitable expert knowledgebase is available. Hence, the proposed Correlation algorithm can adapt to any NID technique and detection algorithm, independent of network traffic and EK data.

Thus, we say that the Correlation model is generalized over network traffic dataset $D$, expert knowledge $E$, intrusion detection technique ID, and detection algorithm A. Therefore,

$$
\text { Correlation : } \mathcal{G}(\mathrm{D}, \mathrm{E}, \mathrm{ID}, \mathrm{A})
$$


Similarly, the proposed Correlated ID metrics work with the predictions of any NID technique and detection algorithm of any dataset and the corresponding EK. Therefore,

$$
\text { Correlated ID Metrics : } \mathcal{G}(\mathrm{D}, \mathrm{E}, \mathrm{ID}, \mathrm{A})
$$

\section{Experimental Analysis and Discussion}

Firstly, we prepare the dataset for classification, then perform misuse detection using the SVM classifier algorithm on the complete dataset. We then correlate the misclassified alerts from SVM through the Correlation algorithm (SVMCorr) in comparison with [9] to obtain the correlated results with minimized false alerts.

Secondly, we perform comparative binary and multiclass classifications with SVM and SVM-Corr machine learning and MLP and MLP-Corr shallow neural network algorithms by splitting the dataset into Normal and four attack classes (DoS, DDoS, Recon, and Theft). We choose this setting to distribute the attack-wise trained classifier models across the HetIoT according to each subnet's specific threat (attack) contexts.

The experimental setup is according to the specifications in the scikit-learn ${ }^{1}$ machine learning library.

\subsection{Experimental Environment}

We list the experimental settings - including the hardware specifications and the algorithmic parameters used for the work in Table 5.

Table 5 Experimental environment settings for misuse detection

\begin{tabular}{ll}
\hline Parameter & Specification \\
\hline CPU & AMD with 6 cores $(3.0 \mathrm{GHz})$ \\
RAM & $24 \mathrm{~GB}(3200 \mathrm{MHz})$ \\
Detection algorithms & SVM, SVM-Corr \\
SVM settings & LinearSVC; cv=4; max_iter=100,000 \\
& solver=sgd; activation=relu; \\
MLP settings & alpha=0.0001; random_state $=1 ;$ \\
& hidden_layer_sizes= $(5,2)$ \\
\hline
\end{tabular}

\subsection{Dataset Preprocessing}

We preprocess the 10-best features CSV file of the Bot-IoT dataset using the Label and One-hot encoding techniques to convert all the categorical features like protocol, ip address, port, and class label into numericals for two types of binary classification:

\footnotetext{
${ }^{1}$ https://scikit-learn.org/stable/
} 
- Normal vs. Attack

- Normal vs. $\{$ DoS, DDoS, Reconnaissance, Theft

\subsubsection{Dataset Normalization}

We scale the encoded dataset features for classification. First, the technique standardizes the feature instances by removing the mean and scaling them to unit variance, as required by the SVM algorithm.

\subsection{Results and Analysis}

We present the details of the experiments conducted and the results obtained, supporting our hypotheses in previous sections.

\subsubsection{SVM-Corr Classifier}

The SVM-Corr classifier makes better predictions with lower false alarm rates, improving classification performance.

We experiment to evaluate the proposed approach's performance compared to the existing work [9] by setting up the Linear SVM classifier with 4-fold cross-validation and max iterations set to 100,000 (for convergence). However, in both cases, the classifier suffered from longer training times (in the order of $10^{3}$ seconds). Table 6 presents the results.

Table 6 SVM and SVM-Corr classifier performances (in \%) on the complete dataset (Normal vs. Attack with $\mathrm{CV}=4$ )

\begin{tabular}{lll}
\hline Classifier & Metric & Result (\%) \\
\hline SVM & Accuracy & 88.3727 \\
SVM-Corr & $\mathcal{A}$ & $\mathbf{9 9 . 9 9 5 1}$ \\
SVM & Precision & $\mathbf{1 0 0}$ \\
SVM-Corr & $\mathcal{P}$ & $\mathbf{9 9 . 9 9 7 4}$ \\
SVM & Recall & 88.3712 \\
SVM-Corr & $\mathcal{R}$ & $\mathbf{9 9 . 9 7 4 0}$ \\
\hline
\end{tabular}

We split the Bot-IoT dataset based on the existing attack types and present the corresponding class-wise (Normal and 4 Attacks) distribution in Table 7.

Table 7 Class-wise Bot-IoT traffic samples distribution

\begin{tabular}{lll}
\hline Class & Train set & Test set \\
\hline Normal & 370 & 107 \\
DoS & 1320148 & 330112 \\
DDoS & 1541315 & 385309 \\
Reconnaissance & 72919 & 18163 \\
Theft & 65 & 14 \\
\hline
\end{tabular}


Table 8 presents the comparative performances of the SVM and SVMCorr classifiers on the attack-wise distributed imbalanced dataset - indicating improved performance. The poor performance of SVM on the Theft attack set is due to very small number of available samples, causing the classifier to not generalize efficiently. The attack-wise training times are of SVM and SVMCorr are of the same order for DoS, DDoS, and Recon attacks - supporting the hypothesis in Corollary 1 and significantly increases for Theft samples - as hypothesized in Theorem 1.

The corresponding confusion matrices in Table 9 indicate the bias of Normal distribution towards class with majority samples, resulting in higher false positives for DoS, DDoS, and Recon and higher false negatives for Theft samples (in red) and the resulting inconsistencies (in blue) despite having good performance scores. However, by incorporating the proposed Correlation model, the SVM performs consistently better with reduced false alerts.

Table 8 Attack-wise SVM and SVM-Corr classifier performances (in \%) on the imbalanced dataset from Table 7 with training time (in seconds)

\begin{tabular}{|c|c|c|c|c|c|c|}
\hline Classifier & Attack & Accuracy & Precision & Recall & F1-score & $\begin{array}{l}\text { Training } \\
\text { Time }\end{array}$ \\
\hline & DoS & 99.9821 & 99.9879 & 99.9942 & 99.9911 & 27.009 \\
\hline \multirow[t]{5}{*}{ SVM } & DDoS & 97.8755 & 99.9920 & 97.8829 & 98.9262 & 107.723 \\
\hline & Recon & 98.4925 & 99.9832 & 98.5089 & 99.2405 & 2.277 \\
\hline & Theft & 19.7917 & 100 & 19.7917 & 33.0435 & 0.012 \\
\hline & & $\mathcal{A}$ & $\mathcal{P}$ & $\mathcal{R}$ & $\mathcal{F}$ & \\
\hline & DoS & 99.9985 & 99.9994 & 99.9376 & 99.9997 & 28.608 \\
\hline \multirow[t]{3}{*}{ SVM-Corr } & DDoS & 99.9979 & 99.9997 & 99.8104 & 99.9998 & 110.704 \\
\hline & Recon & 99.9834 & 99.9945 & 100 & 99.9972 & 2.924 \\
\hline & Theft & 100 & 100 & 100 & 100 & 0.334 \\
\hline
\end{tabular}

Table 9 Attack-wise binary confusion matrices of SVM and SVM-Corr classifiers from Table 8

\begin{tabular}{llllll}
\hline Classifier & Attack & TN & FP & FN & TP \\
\hline \multirow{4}{*}{ SVM } & DoS & 0.05 & 0.95 & 0.0 & 1.0 \\
& DDoS & 0.03 & 0.97 & 0.02 & 0.98 \\
& Recon & 0.0 & 1.0 & 0.01 & 0.99 \\
& Theft & 0.0 & 0.0 & 0.8 & 0.2 \\
\hline \multirow{4}{*}{ SVM-Corr } & CTN & FP & FN & CTP \\
& DoS & 0.95 & 0.05 & 0.01 & 0.99 \\
& Recon & 0.99 & 0.01 & 0.01 & 0.99 \\
& Theft & 1.0 & 0.02 & 0.01 & 0.99 \\
& & 0.0 & 0.0 & 1.0 \\
\hline
\end{tabular}


Table 10 presents the multiclass classification confusion matrices of SVM and SVM-Corr classifiers. We observe the effect of imbalance in the performance scores of Normal and Theft instances by SVM and the improvement offered by the proposed Correlation algorithm in SVM-Corr.

Table 10 Attack-wise multiclass confusion matrices of SVM and SVM-Corr classifiers

\begin{tabular}{|c|c|c|c|c|c|c|c|}
\hline & & & & & Predic & & \\
\hline & & & Normal & DoS & DDoS & Recon & Theft \\
\hline \multirow{6}{*}{ SVM } & \multirow{6}{*}{ Truth } & Normal & 0.41 & 0.2 & 0.3 & 0.06 & 0.03 \\
\hline & & DoS & 0.0 & 0.99 & 0.01 & 0.0 & 0.0 \\
\hline & & DDoS & 0.0 & 0.01 & 0.99 & 0.0 & 0.0 \\
\hline & & Recon & 0.01 & 0.03 & 0.03 & 0.93 & 0.0 \\
\hline & & Theft & 0.0 & 0.24 & 0.54 & 0.0 & 0.22 \\
\hline & & & Normal & DoS & DDoS & Recon & Theft \\
\hline \multirow{5}{*}{ SVM-Corr } & \multirow{5}{*}{ Truth } & Normal & 0.97 & 0.01 & 0.02 & 0.0 & 0.0 \\
\hline & & DoS & 0.0 & 0.99 & 0.01 & 0.0 & 0.0 \\
\hline & & DDoS & 0.0 & 0.01 & 0.99 & 0.0 & 0.0 \\
\hline & & Recon & 0.0 & 0.0 & 0.0 & 1.0 & 0.0 \\
\hline & & Theft & 0.0 & 0.0 & 0.0 & 0.0 & 1.0 \\
\hline
\end{tabular}

\subsubsection{MLP-Corr Classifier}

We choose a shallow neural network classifier to demonstrate the generalizability of the proposed RCA EK framework. We compare the binary classification performances of MLP and MLP-Corr algorithms in Table 11 and present the corresponding confusion matrices in Table 12, observing similar behaviors for SVM and MLP algorithms.

Table 11 Attack-wise MLP and MLP-Corr classifier performances (in \%) on the imbalanced dataset from Table 7 with training time (in seconds)

\begin{tabular}{|c|c|c|c|c|c|c|}
\hline Classifier & Attack & Accuracy & Precision & Recall & F1-score & $\begin{array}{l}\text { Training } \\
\text { Time }\end{array}$ \\
\hline \multirow{5}{*}{ MLP } & DoS & 99.9873 & 99.9873 & 100 & 99.9936 & 41.433 \\
\hline & DDoS & 99.9818 & 99.9857 & 99.9961 & 99.9909 & 50.847 \\
\hline & Recon & 98.5864 & 99.9832 & 98.6028 & 99.2882 & 7.597 \\
\hline & Theft & 11.4583 & 100 & 11.4583 & 20.5607 & 0.233 \\
\hline & & $\mathcal{A}$ & $\mathcal{P}$ & $\mathcal{R}$ & $\mathcal{F}$ & \\
\hline \multirow{4}{*}{ MLP-Corr } & DoS & 99.9956 & 99.9974 & 99.9437 & 99.9988 & 43.138 \\
\hline & DDoS & 99.9979 & 99.9993 & 99.7648 & 99.9934 & 53.055 \\
\hline & Recon & 99.8990 & 99.9945 & 100 & 99.9792 & 8.304 \\
\hline & Theft & 100 & 100 & 100 & 100 & 0.46 \\
\hline
\end{tabular}


Table 12 Attack-wise confusion matrices of MLP and MLP-Corr classifiers from Table 10

\begin{tabular}{llllll}
\hline Classifier & Attack & TN & FP & FN & TP \\
\hline \multirow{4}{*}{ MLP } & DoS & 0.0 & 1.0 & 0.0 & 1.0 \\
& DDoS & 0.11 & 0.89 & 0.0 & 1.0 \\
& Recon & 0.0 & 1.0 & 0.01 & 0.99 \\
& Theft & 0.0 & 0.0 & 0.89 & 0.11 \\
\hline \multirow{4}{*}{ MLP-Corr } & CTN & FP & FN & CTP \\
& DoS & 0.97 & 0.03 & 0.01 & 0.99 \\
& Recon & 0.99 & 0.01 & 0.02 & 0.98 \\
& Theft & 1.0 & 0.02 & 0.02 & 0.98 \\
& & 0.0 & 0.0 & 1.0 \\
\hline
\end{tabular}

The MLP and MLP-Corr multiclass classification confusion matrices in Table 13 demonstrates similar behavior as SVM and SVM-Corr.

Table 13 Attack-wise multiclass confusion matrices of MLP and MLP-Corr classifiers

\begin{tabular}{|c|c|c|c|c|c|c|c|}
\hline & & & & & Predic & & \\
\hline & & & Normal & DoS & DDoS & Recon & Theft \\
\hline \multirow{6}{*}{ MLP } & \multirow{6}{*}{ Truth } & Normal & 0.32 & 0.25 & 0.34 & 0.05 & 0.04 \\
\hline & & DoS & 0.0 & 0.99 & 0.01 & 0.0 & 0.0 \\
\hline & & DDoS & 0.0 & 0.01 & 0.99 & 0.0 & 0.0 \\
\hline & & Recon & 0.02 & 0.05 & 0.06 & 0.87 & 0.0 \\
\hline & & Theft & 0.0 & 0.28 & 0.6 & 0.0 & 0.12 \\
\hline & & & Normal & DoS & DDoS & Recon & Theft \\
\hline \multirow{5}{*}{ MLP-Corr } & \multirow{5}{*}{ Truth } & Normal & 0.98 & 0.01 & 0.01 & 0.0 & 0.0 \\
\hline & & DoS & 0.0 & 0.99 & 0.01 & 0.0 & 0.0 \\
\hline & & DDoS & 0.0 & 0.01 & 0.99 & 0.0 & 0.0 \\
\hline & & Recon & 0.0 & 0.0 & 0.0 & 1.0 & 0.0 \\
\hline & & Theft & 0.0 & 0.0 & 0.0 & 0.0 & 1.0 \\
\hline
\end{tabular}

Finally, we provide a comparative analysis of the proposed work with the existing NIDS schemes in Table 14. The proposed techniques perform better compared to the existing ones upon considering the attack-wise performance scores and training times together.

\subsection{Observations}

We provide few instances connecting the key information from the RCA EK with the misclassified misuse detection alerts, as demonstrated in Algorithm 2. Similarly, we correlate the EK for all the other misclassifications. 
$\stackrel{0}{\mathrm{E}-1}$

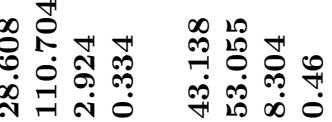

쇼용ำ

$\infty$ ฟै พิ

oे 0

今ं

占

के

เิ

ஓं คㅇㅇㅇ

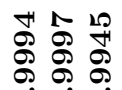

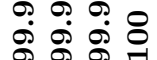

길ำ ஓे के \&
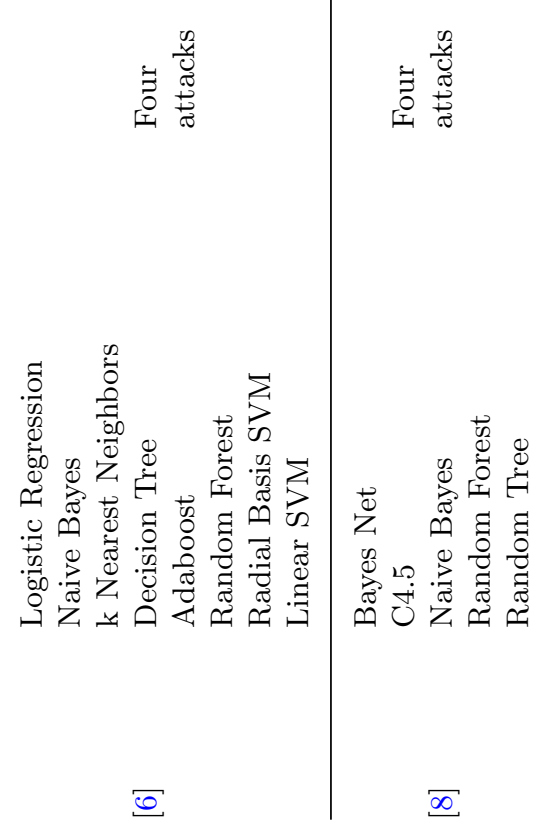

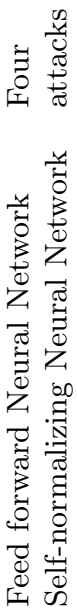

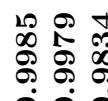
வे

웅요 용

28

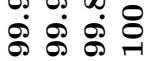

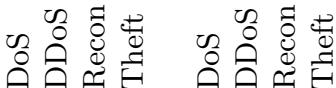

$\sum_{\substack{1 \\ 0}}^{1}$
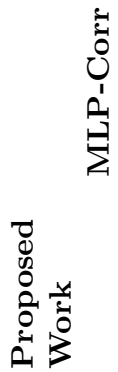
- The traffic instance "2071" in the DoS Test dataset turned out to be a misclassification. The high-level DoS behavior implies that the destination IP receives a large volume of traffic, characterized by the "N_IN_Conn_P_DstIP" feature in the dataset. However, the feature had a very low value, which indicates that it is a false positive.

- The misclassified traffic instance "2" in the Theft Test dataset is a false negative. The corresponding high-level expert knowledge hints that the traffic pattern for information theft closely resembles the Normal traffic, necessitating traffic analysis - resulting in Theft.

We make the following observations from the above information:

- The EK framework minimizes log file analysis.

- Since the size of the EK entries are much lower than the dataset size, it facilitates near real-time operation, as demonstrated in the algorithm analysis.

\section{Conclusion}

We present an RCA EK correlated NIDS evaluation framework generalizable across multiple NID techniques, algorithms, datasets, and corresponding EK. Furthermore, this framework results in training times closer to the SVM and MLP algorithms with minimal computational overhead. It also provides improved classification scores on imbalanced datasets due to contextawareness.

In our future work, we attempt to qualify the impact of attack alerts in a HetIoT threat context by defining a Fault Score metric for the RCA table for alert prioritization and threat (attack) severity assessment. Furthermore, we investigate the bias of Normal distribution and propose a class imbalance invariant data preprocessing technique, which is necessary for online (realtime) threat assessment with smaller number of network traffic samples. We also intend to experiment on different attacks from multiple datasets.

\section{Acknowledgment}

The proposed work is carried out under the 'Development Program of ETU "LETI" within the framework of the Program of Strategic Academic Leadership, Priority-2030'. 


\section{Declarations}

\section{Funding}

Not applicable

\section{Conflicts of interest/Competing interests}

The authors declare that they have no conflict of interest.

\section{Availability of data and material}

The additional data required for the proposed work is presented in Appendix A.

\section{Code availability}

Custom code

\section{Authors' contributions}

Conceptualization, S.P.S.P. and N.A.; methodology, N.A. and S.P.S.P.; software, N.A. and S.P.S.P.; validation, N.A., S.P.S.P. and H.J.; formal analysis N.A. and S.P.S.P.; investigation, N.A., S.P.S.P. and H.J; resources, N.A., S.P.S.P and H.J.; data curation, N.A. and S.P.S.P.; writing - original draft preparation, N.A. and S.P.S.P.; writing - review and editing, N.A., S.P.S.P., H.J., and K.K.; visualization, K.K. and S.P.S.P.; supervision, K.K., H.J., and S.P.S.P.; project administration, K.K. and H.J.

All authors have read and agreed to the published version of the manuscript.

\section{Ethics approval}

Not applicable

\section{Consent to participate}

Agreed without objections

\section{Consent for publication}

Agreed without objections 


\section{References}

[1] Steenwinckel, B, Paepe, D. D., Hautte, S. V., Heyvaert, P., Bentefrit, M., Moens, P., Dimou, A., Bousche, B. V. D., Turck, F. D., Hoecke, S. V., Ongenae, F.: FLAGS: A methodology for adaptive anomaly detection and root cause analysis on sensor data streams by fusing expert knowledge with machine learning. Future Generation Computer Systems. vol. 116, pp. 30-48 (2021)

[2] Alkahtani, H., Aldhyani, T. H. H.: Botnet Attack Detection by Using CNN-LSTM Model for Internet of Things Applications. Security and Communication Networks (2021)

[3] Nikoloudakis, Y., Kefaloukos, I., Klados, S., Panagiotakis, S., Pallis, E., Skianis, C., Markakis, E. K.: Towards a Machine Learning Based Situational Awareness Framework for Cybersecurity: An SDN Implementation. Sensors. 21, 4939 (2021)

[4] Alqahtani, M., Mathkour, H., Ismail, M. M. B.: IoT Botnet Attack Detection Based on Optimized Extreme Gradient Boosting and Feature Selection. Sensors. 6336, 20 (2020)

[5] Da Silva, D. M. A., Sofia, R. C.: A Discussion on Context-Awareness to Better Support the IoT Cloud/Edge Continuum. IEEE Access. vol. 8, pp. 193686-193694 (2020)

[6] Sriram, S., Vinaykumar, R., Alazab, M., Soman, K. P.: Network Flow based IoT Botnet Attack Detection using Deep Learning. IEEE INFOCOM 2020 - IEEE Conference on Computer Communications Workshops (INFOCOM WKSHPS). pp. 189-194 (2020)

[7] Zheng, D., Hong, Z., Wang, N., Chen, P.: An Improved LDA-Based ELM Classification for Intrusion Detection Algorithm in IoT Application. Sensors. 1706, 20 (2020)

[8] Shafiq, M., Tian, Z., Sun, Y., Du, X., Guizani, M.: Selection of effective machine learning algorithm and Bot-IoT attacks traffic identification for internet of things in smart city. Future Generation Computer Systems. vol. 107, pp. 433-442 (2020)

[9] Koroniotis, N., Moustafa, N., Sitnikova, E., Turnbull, B.: Towards the development of realistic botnet dataset in the Internet of Things for network forensic analytics: Bot-IoT dataset. Future Generation Computer Systems. vol. 100, pp. 779-796 (2019)

[10] Zhang, H., Yu, X., Ren, P.: Deep Adversarial Learning in Intrusion Detection: A Data Augmentation Enhanced Framework. Preprint at https: 
//arxiv.org/pdf/1901.07949.pdf (2019)

[11] Trnka, M., Svacina, J., Cerny, T., Song, E., Hong, J., Bures, M.: Securing Internet of Things Devices Using the Network Context. IEEE Transactions on Industrial Informatics. vol. 16(6), pp. 4017-4027 (2019)

[12] Ibitoye, O., Shafiq, O., Matrawy, A.: Analyzing Adversarial Attacks Against Deep Learning for Intrusion Detection in IoT Networks. IEEE Global Communication Conference, (2019)

[13] Qiu, T., Chen, N., Li, K., Atiquzzaman, M., Zhao, W.: How Can Heterogeneous Internet of Things Build Our Future: A Survey. IEEE Communications Surveys \& Tutorials. vol. 20, pp. 2011-2027 (2018)

[14] Doshi, R., Apthorpe, N., Feamster, N.: Machine Learning DDoS Detection for Consumer Internet of Things Devices, IEEE Symposium on Security and Privacy Workshops (2018)

[15] Sezer, O. B., Dogdu, E., Ozbayoglu, M.: Context Aware Computing, Learning and Big Data in Internet of Things: A Survey. IEEE Internet of Things Journal. vol. 5(1), pp. 1-27 (2018)

[16] Xu, G., Cao, Y., Ren, Y., Li X., Feng, Z.: Network security situation awareness based on semantic ontology and user-defined rules for Internet of Things. IEEE Access. vol. 5, pp. 21046-21056 (2017)

[17] Behal, S., Kumar, K.: Detection DDoS Attacks and Flash Events using Information Theory Metrics - An Empirical Investigation. Computer Communications (2017)

[18] Kolias, C., Kambourakis, G., Stavrou, A., Voas, J.: DDoS in the IoT: Mirai and Other Botnets, Computer. vol. 50, pp. 80-84 (2017)

[19] Hoque, N., Bhuyan M. H., Baishya R. C., Bhattacharyya, D. K., Kalita, J.K.: Network Attacks: Taxonomy, Tools and Systems. Journal of Network and Computer Applications. pp. 307-324 (2014)

[20] Jesudoss, A., Subramaniam, N. P.: A Survey on Authentication Attacks and Countermeasures in a Distributed Environment. Indian Journal of Computer Science and Engineering (IJCSE) (2014)

[21] Zargar, S. T., Joshi, J., Tipper, D.: A Survey of Defense Mechanisms Against Distributed Denial of Service (DDoS) Flooding Attacks. IEEE Communications Surveys \& Tutorials (2013)

[22] Hoque, M. S., Mukit, M. A., Bikas, M. A. N.: An Implementation of Intrusion Detection System using Genetic Algorithm. International Journal of 
Network Security \& Its Applications (IJNSA). vol. 4, no, 2 (2012)

[23] Paliwal, S., Gupta, R.: Denial-of-Service, Probing \& Remote to User (R2L) Attack Detection using Genetic Algorithm. International Journal of Computer Applications. vol. 60, no. 19, pp. 57-62 (2012)

[24] Tankard, C.: Persistent threats and how to monitor and deter them. Network Security, pp. 16-19 (2011)

[25] Al-Mamory, S. O., Zhang, H.: Intrusion detection alarms reduction using root cause analysis and clustering. Computer Communications. vol. 32, pp. 419-430 (2009)

\section{Appendix A Expert Knowledgebase}

In this section, we provide the detailed version of the RCA expert knowledgebase, $\left(E_{R C A}\right)$ divided attack-wise. The setting presented, reduces search time based on the attack type - resulting in lower training time.

The framework contains the RCA EK of the Bot-IoT dataset [9] in Tables A1 through A4, offering a list of possible faults for an attack category compiled by domain experts, where an attack is associated with a high-level unexpected network behavior. We extract the information from the resources [14]-[24] mentioned in [9]. In addition, the Bot-IoT NID dataset offers information about such behaviors - identified as specific faults and their probable causes that include system vulnerabilities. We use this information obtained from multiple repositories as the EK to correlate ID alerts. 
22 Expert Knowledge Correlated IDS Evaluation Framework for HetIoT

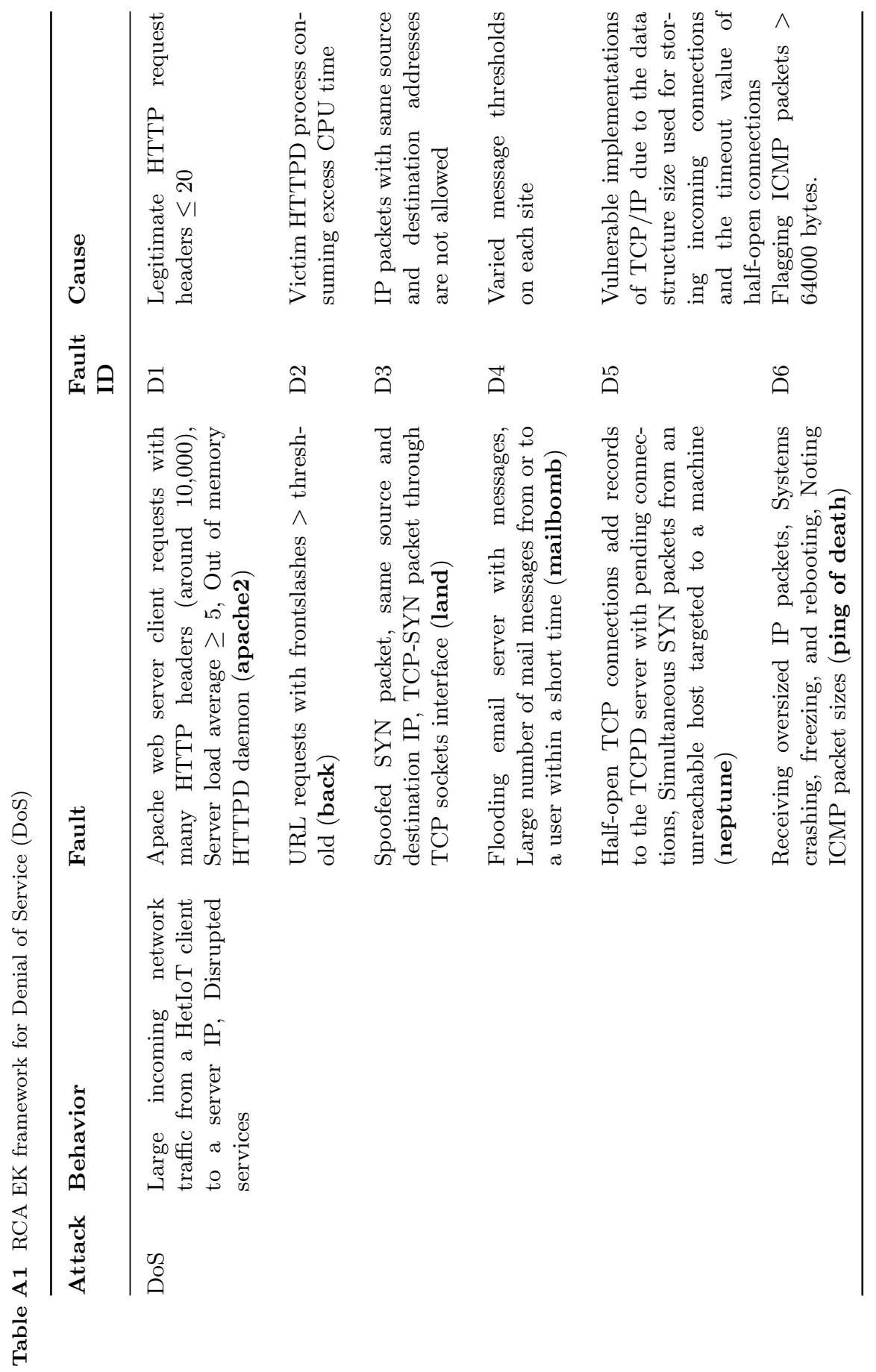




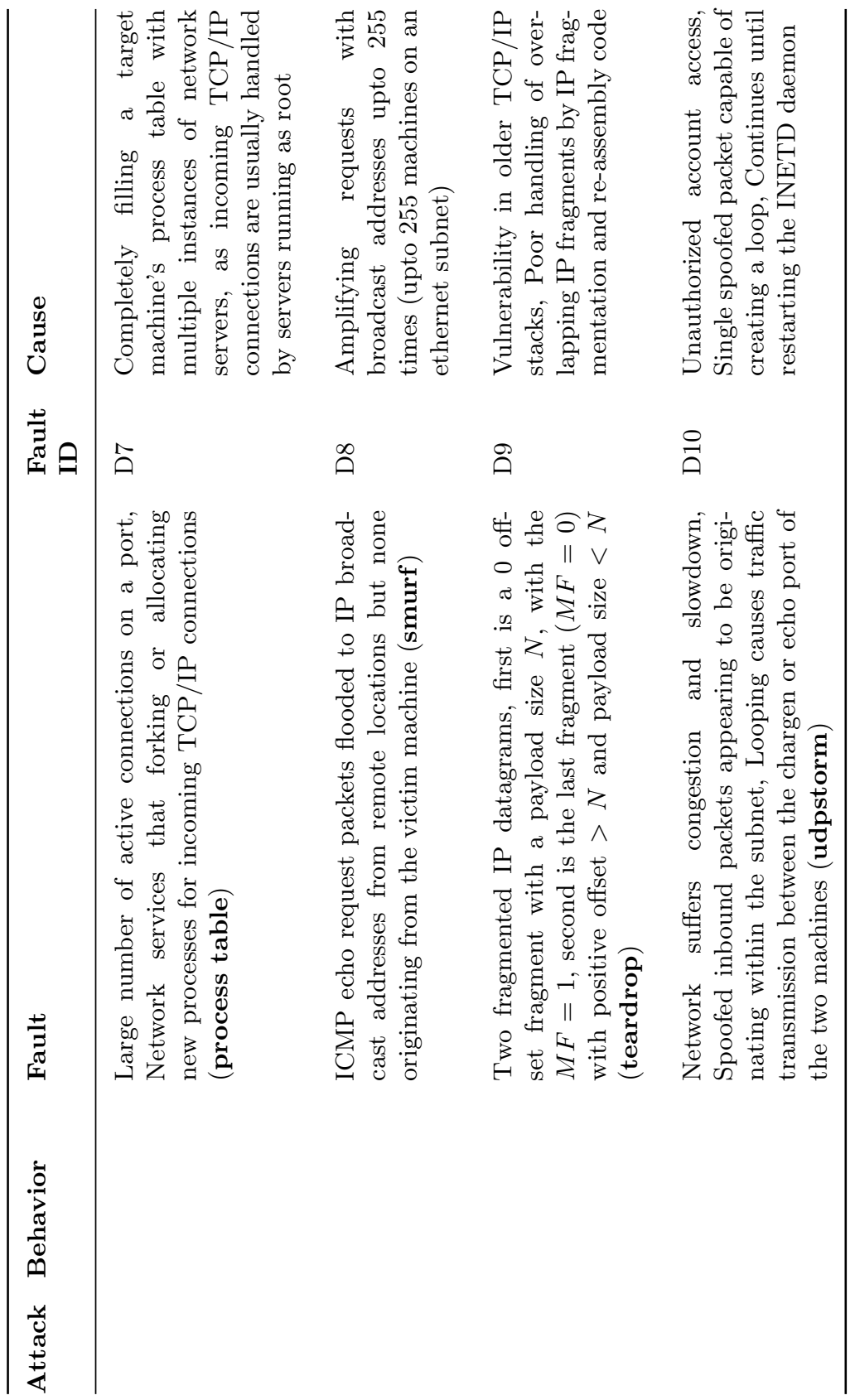


24 Expert Knowledge Correlated IDS Evaluation Framework for HetIoT

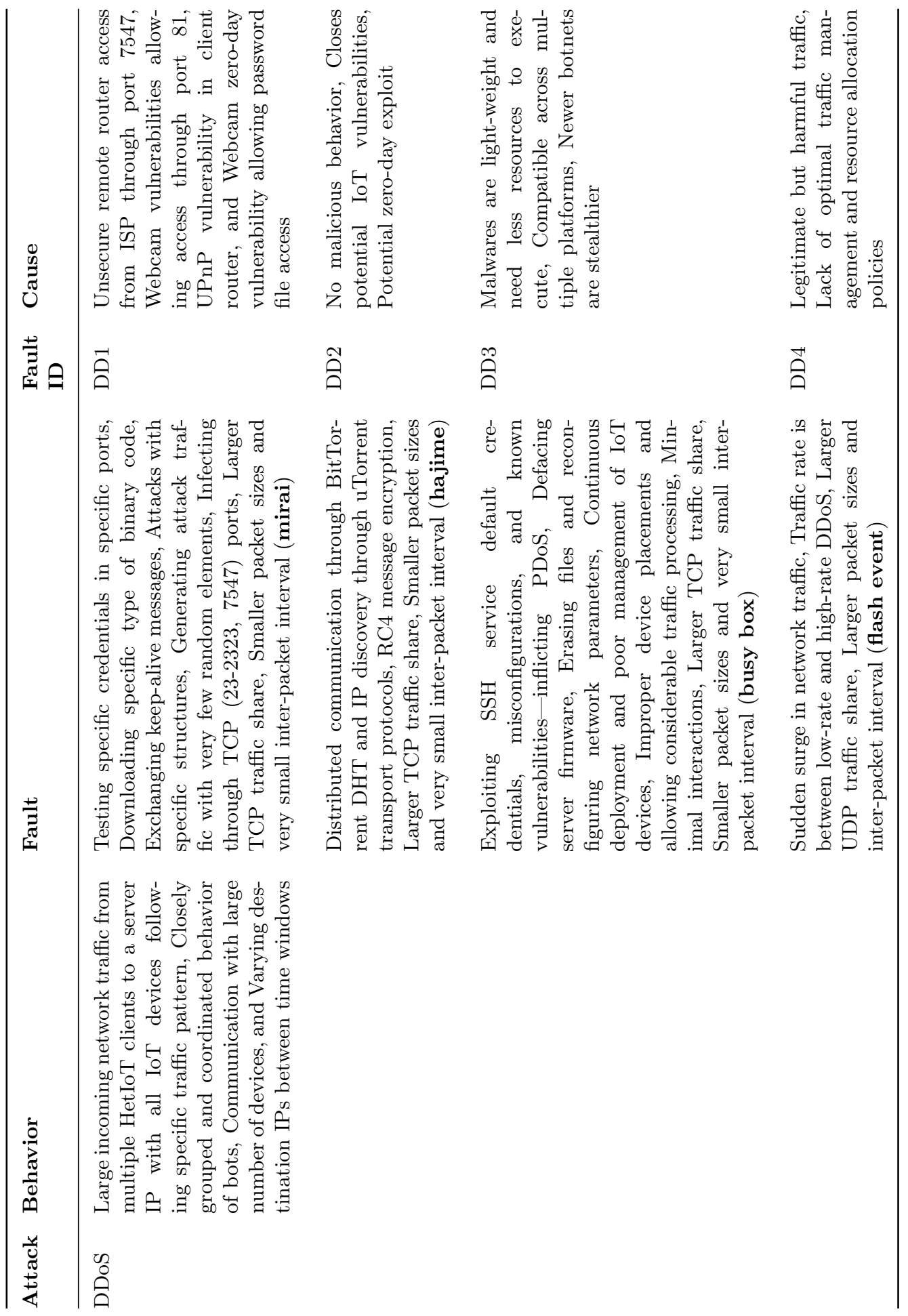




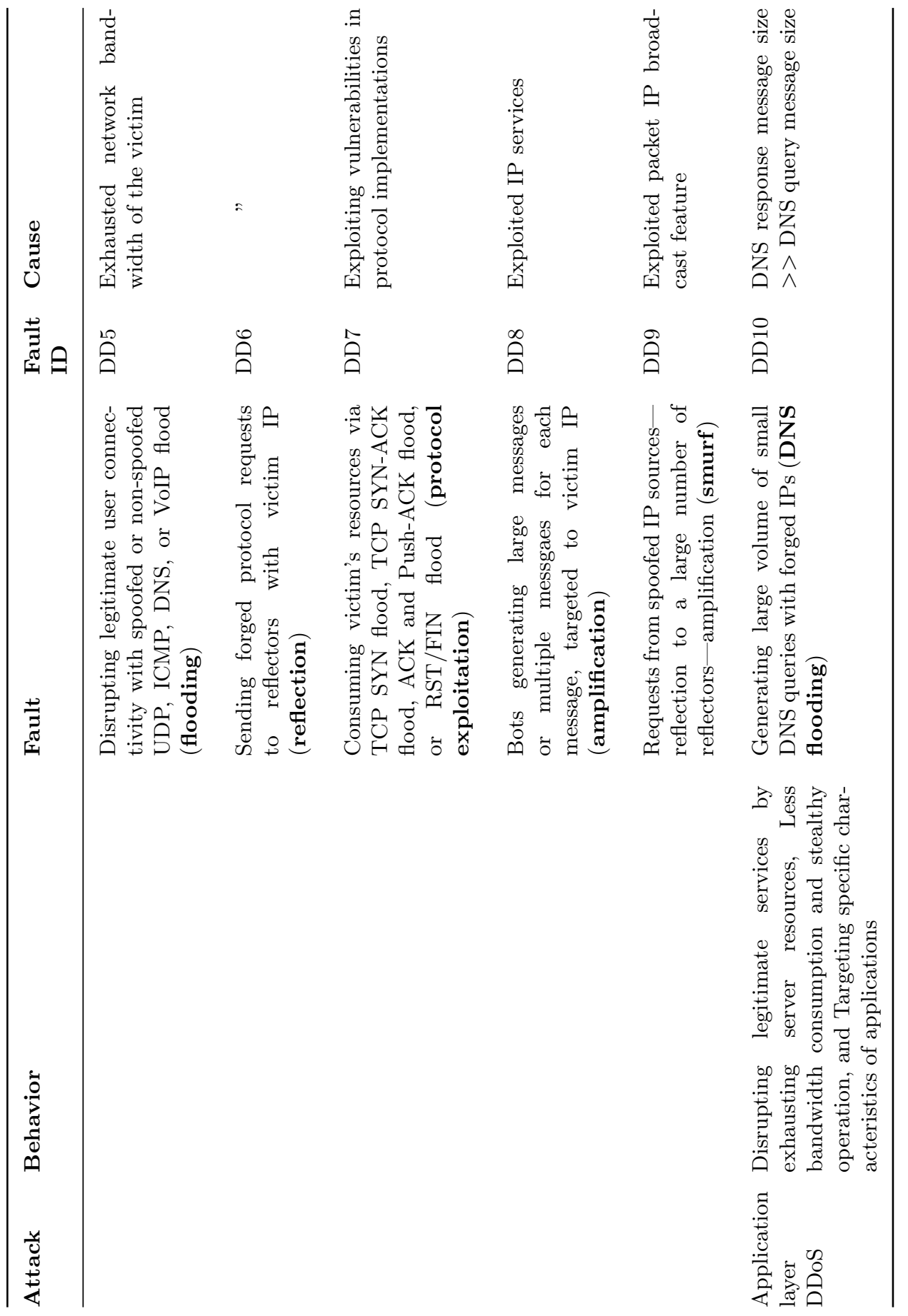




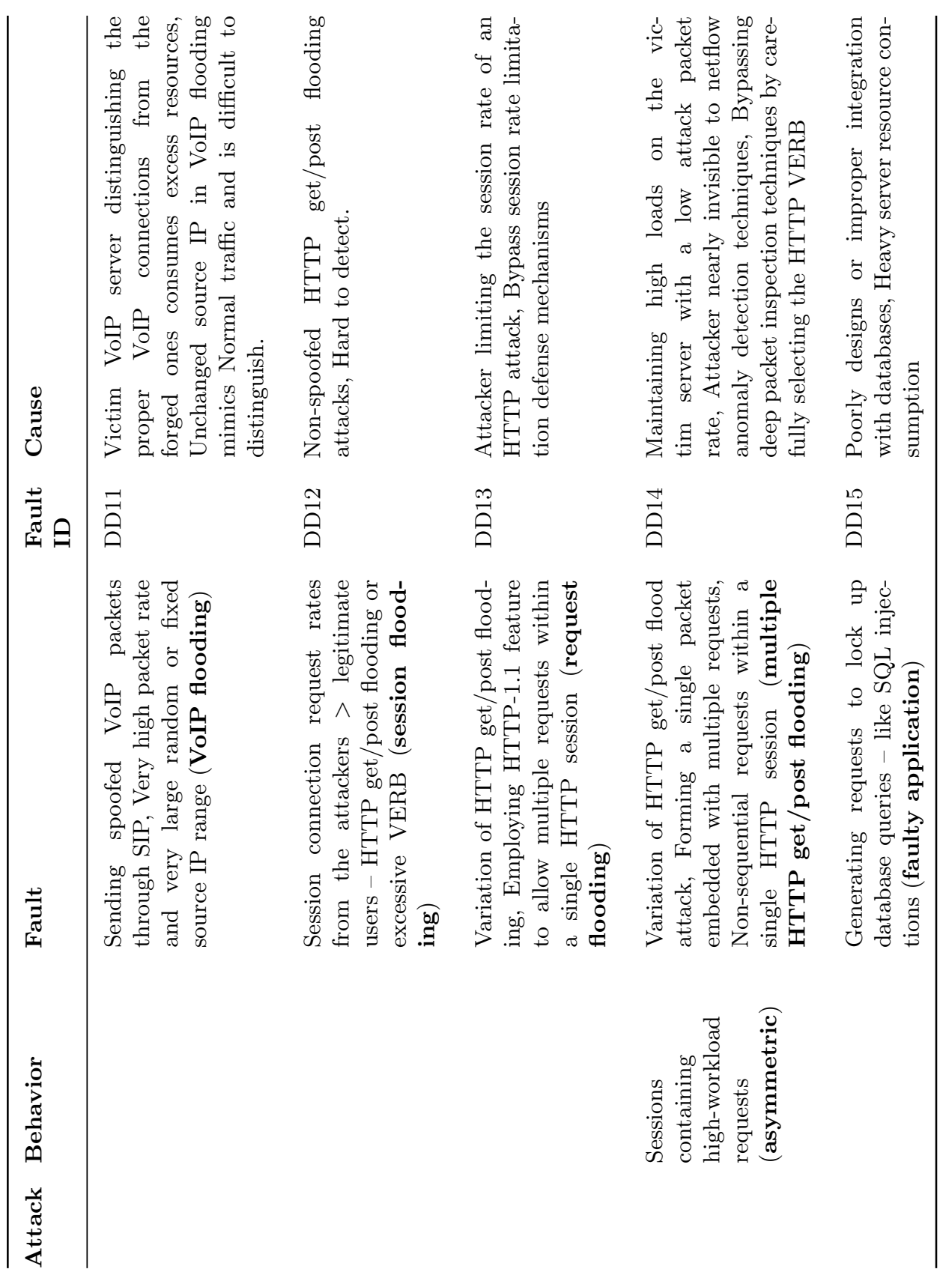


Expert Knowledge Correlated IDS Evaluation Framework for HetIoT

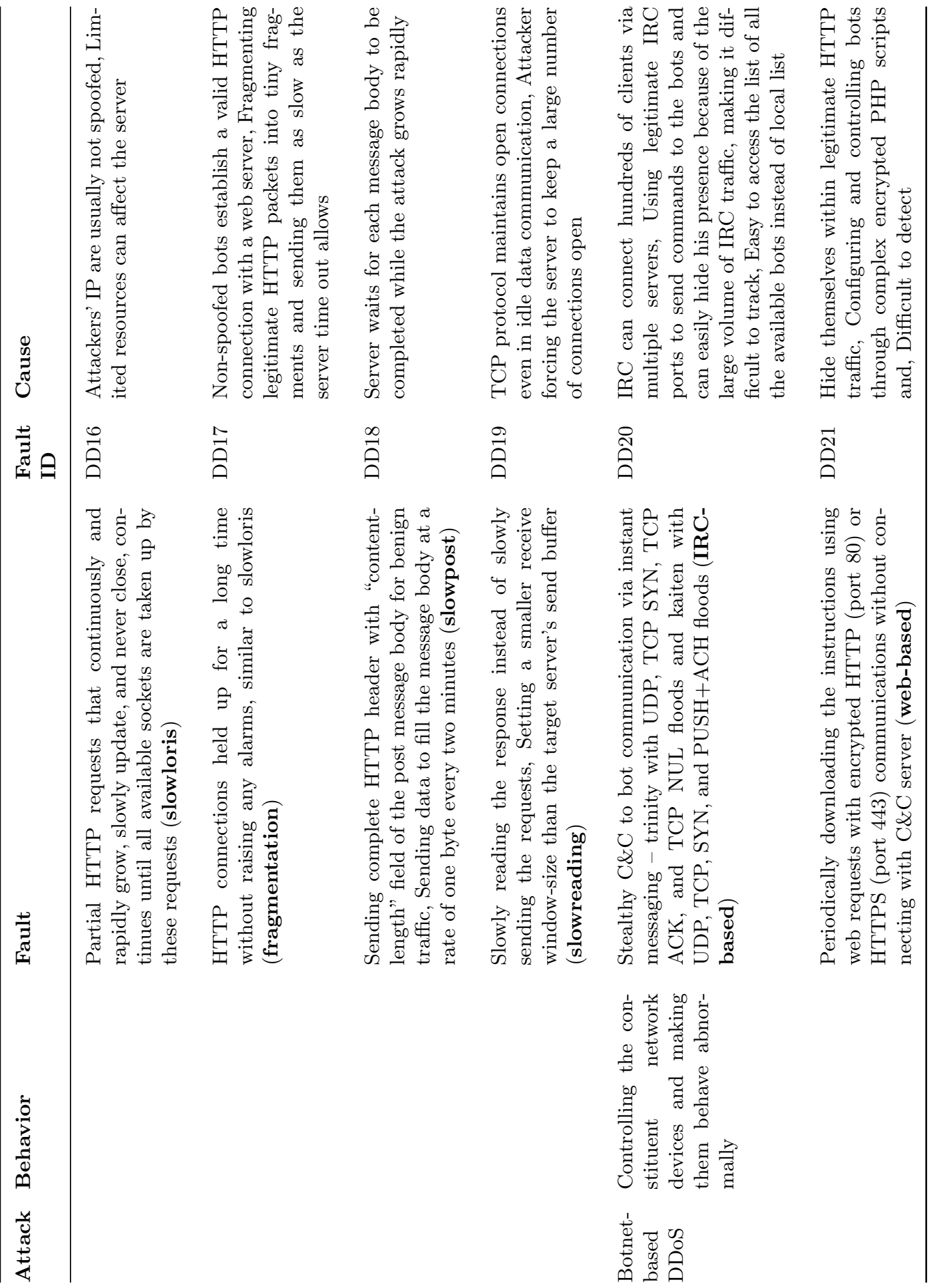




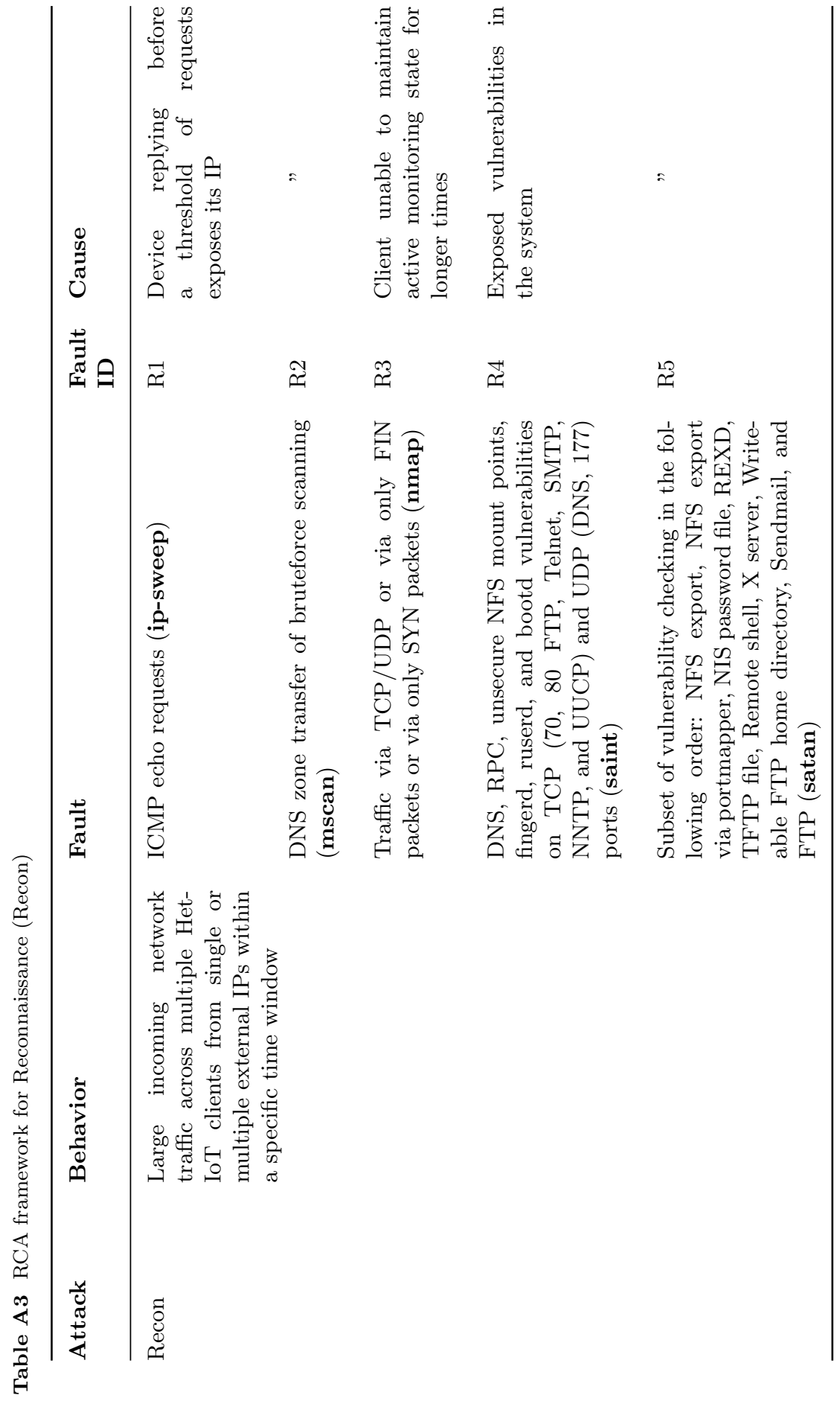




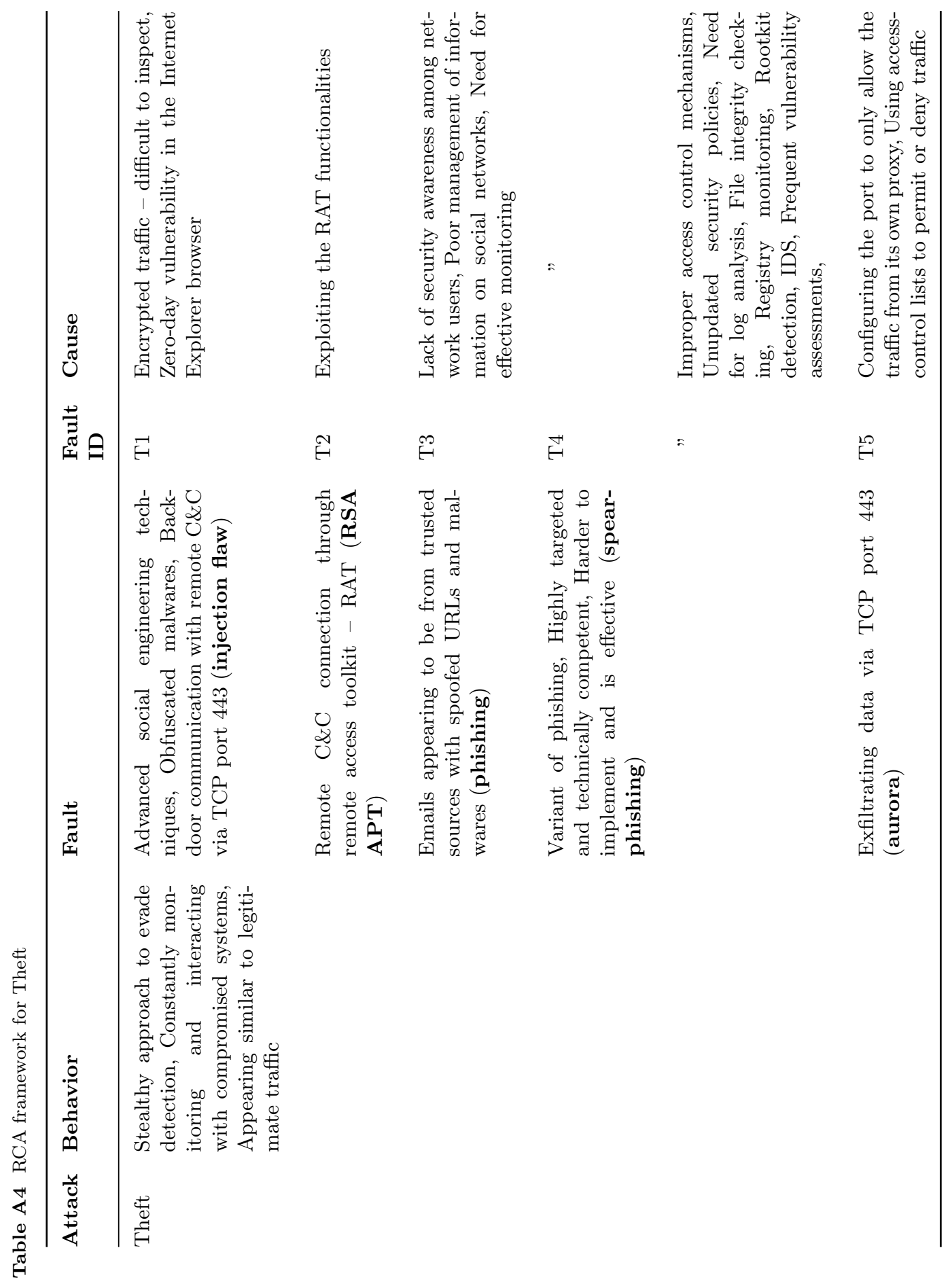




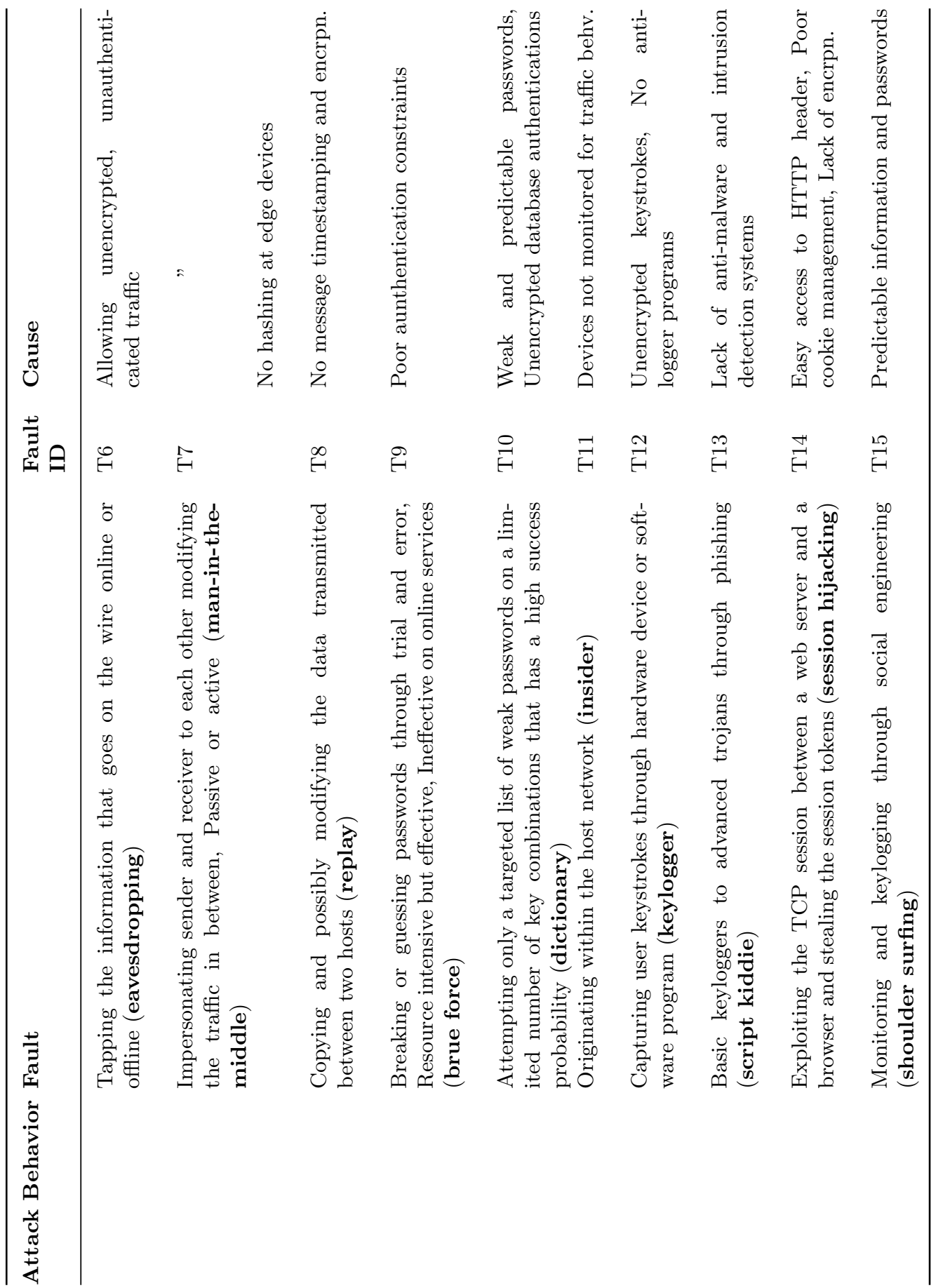

